\title{
Noninvasive Quantification of Liver Fat Content by Different Gradient Echo Magnetic Resonance Imaging Sequences in Patients with Nonalcoholic Fatty Liver Disease
}

\begin{abstract}
Background: Noninvasive quantification of liver fat by gradient echo (GRE) technique is an interesting issue in quantitative magnetic resonance imaging. In this study, the fat content in patients with nonalcoholic fatty liver disease (NAFLD) was quantified with GRE sequences with different $T_{1}$ and $T_{2}^{*}$ weighting. Methods: This prospective, cross-sectional study was performed on thirty NAFLD patients. Sixteen GRE sequences with different $T_{1}$ weighting were performed with four echo times. In each sequence, repetition time (TR) or flip angle was changed and other parameters were fixed. Forty-eight fat indexes (FIs) from 16 sequences were calculated based on three methods. To determine the relationship between FIs and histological findings, Pearson's correlation coefficient was used at the level of $1 \%$ significance. Results: Mean FIs which obtained from Eq. 3 have the maximum values in comparison to other FIs. The maximum FI was $23.58 \%$, which related to heavily $T_{1}$ weighted sequence obtained with method 3. The minimum FI was $-2.49 \%$, which related to the minimal $T_{1}$ weighted obtained with method 2 . FIs increase with a flip angle, especially at low flip angles. Increase the TR parameter decrease the FIs gradually. Calculated FIs with methods 1 and 3 stronger correlated with histological findings relative to calculated FIs with method 2. Conclusion: For fat quantification, $T_{1}$ relaxation effects probably more critical than $T_{2}^{*}$. Flip angle parameter could be a major factor causing the overestimation of liver fat content. Sequences with low flip angle are more suitable for fat quantification with methods 1 and 3. In fat quantification with GRE techniques, it is possible that the third and fourth echoes are unnecessary.
\end{abstract}

Keywords: Gradient echo magnetic resonance imaging, nonalcoholic fatty liver disease, $T_{1}$ and $T_{2}{ }^{*}$ relaxation effects

\section{Introduction}

Nonalcoholic fatty liver disease (NAFLD) is the most common chronic liver disorder in Western countries. The prevalence rate of this disease is $20 \%-30 \%$ in the general population and $60 \%-80 \%$ among diabetics and obese patients. ${ }^{[1,2]}$ Hepatic steatosis or accumulation of lipid vacuoles within hepatocytes is the primary histologic hallmark for diagnosis of NAFLD. ${ }^{[3]}$

Noninvasive detection and quantification of the steatosis has a considerable importance in clinical hepatology. In liver surgery, since the presence of moderate or severe steatosis may lead to graft failure in recipients and postponed the recovery process of donors, so the degree of steatosis should be measured accurately for transplant decision-making. ${ }^{[4,5]}$ The steatosis should be carefully determined in NAFLD patients

This is an open access journal, and articles are distributed under the terms of the Creative Commons Attribution-NonCommercial-ShareAlike 4.0 License, which allows others to remix, tweak, and build upon the work non-commercially, as long as appropriate credit is given and the new creations are licensed under the identical terms. who underwent interventional activities such as exercise, diet, and lifestyle changes for judgment about the effectiveness of these treatments. ${ }^{[6]}$ Early diagnosis and treatment of NAFLD can prevent the progression of the disease to more severe conditions such as nonalcoholic steatohepatitis (NASH), cirrhosis, and hepatocellular carcinoma. ${ }^{[7]}$ Liver fat content can be evaluated by biopsy and imaging modalities such as ultrasonography (US), computed tomography, magnetic resonance imaging (MRI), and MR spectroscopy. ${ }^{[8]}$

A biopsy is a current gold standard for assessment of liver fat content; however, due to invasive nature, this method has a number of limitations such as risk of bleeding, infection, and bile leakage. ${ }^{[9]}$ Moreover, this procedure is not reproducible and associated with sampling errors. ${ }^{[10]}$

How to cite this article: Zabihzadeh $M$ Gharibvand MM, Motamedfar A, Tahmasebi M, Sina AH, Bahrami K, et al. Noninvasive quantification of liver fat content by different gradient echo magnetic resonance imaging sequences in patients with nonalcoholic fatty liver disease. J Med Sign Sens 2018;XX:XX-XX.

Received: July, 2018. Accepted: September, 2018.

\section{Mansour \\ Zabihzadeh $^{1,2,3}$, Mohammad Momen Gharibvand4, Azim Motamedfar ${ }^{4}$, Morteza \\ Tahmasebi ${ }^{4}$, Amir Hossein Sina ${ }^{5}$, Kavous Bahrami ${ }^{4}$, Mozafar Naserpour ${ }^{2,6}$}

${ }^{1}$ Research Center for Infectious Diseases of Digestive System, School of Medicine, Ahvaz Jundishapur University of Medical Sciences, ${ }^{2}$ Department of Medical Physics, Faculty of Medicine, Ahvaz Jundishapur University of Medical Sciences, ${ }^{3}$ Department of Clinical Oncology, Golestan Hospital, Ahvaz Jundishapur University of Medical Sciences, ${ }^{4}$ Department of Radiology, School of Medicine, Ahvaz Jundishapur University of Medical Sciences, ${ }^{5}$ Department of Radiology, Ahvaz Jundishapur University of Medical Sciences, Ahvaz, ${ }^{6}$ Department of Radiology Technology, Behbahan Faculty of Medical Sciences, Behbahan, Iran
Address for correspondence: Dr. Mozafar Naserpour, Department of Medical Physics, Faculty of Medicine, Ahvaz Jundishapur University of Medical Sciences, Ahvaz, Iran. Department of Radiology Technology, Behbahan Faculty of Medical Sciences, Behbahan, Iran.

E-mail: naserpour1983@gmail. com

Website: www.jmss.mui.ac.ir DOI: 10.4103/jmss.JMSS_32_18 
Hence, there have been comprehensive efforts to substitute this procedure by noninvasive imaging modalities.

US considered as a rapid and low-cost instrument for assessment of hepatic steatosis whereas this subjective modality suffered from low sensitivity and accuracy. ${ }^{[11,12]}$ Several studies have been reported that good correlation between fat quantification by chemical-shift Gradient echo (GRE) MRI techniques and histopathological findings. ${ }^{[13-15]}$

Despite the numerous advantages of GRE techniques such as rapidity, good accuracy, and ability to perform at different scanners, the main weakness of this technique is that the fat measurement may be biased by $T_{1}$ and $T_{2}{ }^{*}$ relaxation effects. Modifying the imaging parameters that control $T_{1}$ and $T_{2}{ }^{*}$ weighting may lead to miscalculation of liver fat quantity. Although comprehensive research ${ }^{[14-18]}$ has been carried out on quantifying liver fat by GRE MR imaging, only a few studies have attempted to investigate the effects of the imaging parameters that control the $T_{1}$ and $T_{2}{ }^{*}$ weighting on in vivo liver fat quantification. ${ }^{[19-22]}$ Hence, this study was set out to examine the GRE protocols with different $T_{1}$ and $T_{2}{ }^{*}$ weighting for liver fat quantification in patients with NAFLD.

\section{Methods}

\section{Study design and patient population}

This prospective, cross-sectional, single-center study was approved by the local ethical committee. In a period of $>2$ years (from September 2015 to November 2017), 34 adult patients over 18 years with biopsy-confirmed NAFLD in consistent with NASH Clinical Research Network (NASH CRN) ${ }^{[23]}$ were recruited from Golestan Educational Hospital of Ahvaz, Iran, to participate in this research. Exclusion criteria were as follows: patients with pregnancy (none), alcohol consumption (none), use of therapeutic interventions between biopsy and MRI test (3 cases), MRI contraindications (1 case), and known history of other hepatic diseases. The mean time interval from biopsy to MRI examination was 21 days (range $=8-34$ days). The purpose of the study was clearly explained and written informed consent was obtained from all participants.

\section{Liver biopsy and histopathological assessment}

Ultrasound-guided needle biopsies were performed with $18 \mathrm{G}$ from the right lobe of the liver. The mean specimen size was $2 \mathrm{~cm} \times 0.1 \mathrm{~cm}$ fixed in formalin for $24 \mathrm{~h}$, then $0.4 \mu \mathrm{m}$ sections of the tissue stained with hematoxylin and eosin, trichrome, and reticulin. Two hepatic pathologists who were blinded to the MRI reports examined slides according to NASH CRN histological scoring system. ${ }^{[23]}$ Steatosis was classified into four grades: fat deposition in $0 \%-5 \%$ of hepatocytes (Grade 0), 5\%-33\% (Grade 1), 34\%-66\% (Grade 2), and above 67\% (Grade 3).

\section{Magnetic resonance imaging sequences}

All examinations were performed using 1.5 Tesla MR scanner (Essenza, Siemens Medical Systems, Erlangen Germany) equipped with four-channel torso coil. Sixteen GRE sequences with different $T_{1}$ weighting contrast were performed for each patient with four echo times $\left(\mathrm{TE}_{1}=2.31, \mathrm{TE}_{2}=4.86\right.$, $\mathrm{TE}_{3}=7.67$, and $\mathrm{TE}_{4}=10.58 \mathrm{~ms}$ ). In each sequence, three slices with 6-mm thickness, field of view $=380 \mathrm{~mm}$, bandwidth $=500 \frac{\mathrm{Hz}}{\text { Pixel }}$ were performed from the liver at hilarious level. The taken images were included of three first out-of-phase images $\left(\mathrm{OP}_{1}\right)$ for $\mathrm{TE}_{1}$, three first in-phase images $\left(\mathrm{IP}_{1}\right)$ for $\mathrm{TE}_{2}$, three second out-of-phase images $\left(\mathrm{OP}_{2}\right)$ for $\mathrm{TE}_{3}$, and three second in-phase images $\left(\mathrm{IP}_{2}\right)$ for $\mathrm{TE}_{4}$. Therefore, 12 images were achieved from any sequence. In each sequence, repetition time (TR) or flip angle $(\alpha)$ parameter was changed and set out according to Table 1 , and other scan parameters were fixed at all sequences [Table 1].

\section{Image analysis}

All analyzes were performed by two experienced radiologists who were blinded to histopathological findings at the same workstation. To consider the effect of fat inhomogeneity, regions of interest (ROIs) were placed on three slices at 7 different regions of liver parenchyma by avoiding other structures such as diaphragm, major vessels and so on. ROIs which placed on $\mathrm{OP}_{1}$ images were copied into other images identically. The mean signal intensity of $\mathrm{OP}_{1}, \mathrm{IP}_{1}, \mathrm{OP}_{2}$, and $\mathrm{IP}_{2}$ images were measured separately for each sequence by averaging the 21 ROIs.

Calculation of fat indexes by magnetic resonance imaging signals

In this study, fat index (FI) values were measured for all 16 sequences separately with three different methods. Method

Table 1: Characteristics of repetition time (ms) and flip angle ( $\alpha$ : Degree) at different sequences

\begin{tabular}{lcc}
\hline Sequence number & TR & Flip angle $(\boldsymbol{\alpha})$ \\
\hline Sequence 1 & 50 & 20 \\
Sequence 2 & 50 & 45 \\
Sequence 3 & 50 & 70 \\
Sequence 4 & 50 & 90 \\
Sequence 5 & 100 & 20 \\
Sequence 6 & 100 & 45 \\
Sequence 7 & 100 & 70 \\
Sequence 8 & 100 & 90 \\
Sequence 9 & 150 & 20 \\
Sequence 10 & 150 & 45 \\
Sequence 11 & 150 & 70 \\
Sequence 12 & 150 & 90 \\
Sequence 13 & 200 & 20 \\
Sequence 14 & 200 & 45 \\
Sequence 15 & 200 & 70 \\
Sequence 16 & 200 & 90
\end{tabular}

RT: Repetition time 
1: In this method, FIs (1-16) were calculated by considering signal intensity changes from first dual echoes $\left(\mathrm{OP}_{1}\right.$ and $\mathrm{IP}_{1}$ images) according to Eq. 1 for all 16 sequences, respectively. Method 2: FIs values from 17 to 32 were calculated by providing this method. Like the first method and taking into account of the second dual echoes $\left(\mathrm{OP}_{2}\right.$ and $\mathrm{IP}_{2}$ images), FIs (17-32) were achieved by Eq. 2. Method 3: In this method, signal loss due to $T_{2}^{*}$ decay was corrected. Estimated FI by this method was achieved using Eq. 3.

$$
\begin{aligned}
& \left(\frac{\mathrm{SI}_{\mathrm{IP}_{1}}-\mathrm{SI}_{\mathrm{OP}_{1}}}{2 \mathrm{SI}_{\mathrm{IP}_{1}}}\right) \times 100^{[24]} \\
& \left(\frac{\mathrm{SI}_{\mathrm{IP}_{2}}-\mathrm{SI}_{\mathrm{OP}_{2}}}{2 \mathrm{SI}_{\mathrm{IP}_{2}}}\right) \times 100 \\
& \left(\frac{\left(\mathrm{SI}_{\mathrm{IN}_{1}} \exp ^{\left(4.86 / \mathrm{T}_{2}^{*}\right)}-\mathrm{SI}_{\mathrm{OUT}_{1}} \exp ^{\left(2.31 / \mathrm{T}_{2}^{*}\right)}\right)}{2 S I_{I N_{1}} \exp \left(4.86 / T_{2}^{*}\right)}\right) \times 100 \\
& \mathrm{~T} 2 *=\Delta \mathrm{TE} \ln \left(\frac{\mathrm{SI}_{\mathrm{IN}_{1}}}{\mathrm{SI}_{\mathrm{IN}_{2}}}\right)
\end{aligned}
$$

Where $\mathrm{SI}_{\mathrm{OP1}}$ and $\mathrm{SI}_{\mathrm{IP1} 1}$ are out of phase and in phase corresponding to the mean signal intensity of first dual GREs, respectively, $\mathrm{SI}_{\mathrm{OP} 2}$ and $\mathrm{SI}_{\mathrm{IP} 2}$ and are corresponding to mean signal intensity of second dual GREs. $\triangle \mathrm{TE}$ is the time difference between $\mathrm{IP}_{2}$ and $\mathrm{IP}_{1}(10.58-4.86=5.72 \mathrm{~ms})$. In each sequence, three FIs were calculated with three different methods (Eqs. 1-3). Therefore, 48 FIs obtained for each patient normally. As stated in Eq. 1, FIs (1-16) which are related to sequences (1-16) were measured by considering signal intensity changes at first dual echoes. FIs (17-32) established using Eq. 2 from signal intensity changes at second dual echoes of each sequence. Finally, FIs (33-48) were obtained using Eq. 3 and correction of $\mathrm{T}_{2} *$ decay effects for each sequence.

\section{Statistical analysis}

The calculated FIs by MRI are presented as a mean \pm standard deviation. The correlation between histopathologic findings and MRI FIs was performed using Pearson coefficient. Data management and analysis were performed using SPSS 16.0 (SPSS Inc., SPSS for Windows, Chicago, USA). Significance levels were set at the $1 \%$ level.

\section{Results}

\section{Demographic and clinical data}

In this study, MRI sequences were performed for the thirty confirmed NAFLD adult patients (mean age: $41.4 \pm 11.4$ years). Characteristics and clinical data of these patients are given in Table 2 .

\section{The results of calculated fat indexes}

The first set of analysis indicated the impact of variable $T_{1}$ and $T_{2}^{*}$ weighting imaging parameters for calculation of FIs. Totally 48 FIs were calculated using three equations. The results obtained from the calculation of FIs are shown in
Table 3. As can be seen in this table, the mean FIs (17-32) which calculated from Eq. 2 are very low and in some indexes have a negative value. The mean FIs (33-48) which obtained from Eq. 3 have the maximum values in comparison to the other FIs. The maximum mean FI was $23.58 \%$ (FI36) which corresponds to heavily $T_{1}$ weighted pulse sequence (sequence 4 $\mathrm{TR}=50 \mathrm{~ms}, \alpha=90^{\circ}$ quantified by method 3 . The minimum mean FI was $-2.49 \%$ (FI29) which corresponds to the minimal $T_{1}$ weighted pulse sequence (sequence $13 \mathrm{TR}=200 \mathrm{~ms}$, $\alpha=20^{\circ}$ quantified by method 2 [Table 3].

Relationship between flip angle parameter and calculated fat indexes

Figure 1a-c illustrates the relationship between mean calculated FI and flip angles for different TR parameters. As can be seen in these figures, the mean calculated FI increases with rising of flip angle, especially at low flip angles. Mean FI was obtained by extrapolating the curves to flip angle $\rightarrow 0^{\circ}$ for minimizing the effect of this parameter [Figure 1].

Relationship between calculated fat index and repetition

\begin{tabular}{|c|c|}
\hline Characteristics & Result \\
\hline Age (year) & Mean: $41.4 \pm 11.4$ year $(19-62)$ \\
\hline \multicolumn{2}{|l|}{ Gender (\%) } \\
\hline Female & 19 of $30(63.3)$ \\
\hline Male & 11 of $30(36.7)$ \\
\hline BMI $\left(\mathrm{kg} / \mathrm{m}^{2}\right)$ & $29.0 \pm 4,(21.6-36.6)$ \\
\hline \multicolumn{2}{|l|}{ Steatosis grade $(\%)$} \\
\hline $\begin{array}{l}\text { Fat deposited in }<5 \text { of } \\
\text { hepatocytes }\end{array}$ & None $(0)$ \\
\hline 5-33 of hepatocytes & $16(53.3)$ \\
\hline $34-66$ of hepatocytes & $10(33.3)$ \\
\hline$>66 \%$ of hepatocytes & $4(13.3)$ \\
\hline \multicolumn{2}{|l|}{ Lobular inflammation } \\
\hline No foci & $4(13.3)$ \\
\hline$<2$ foci per $200 \times$ field & $19(63.3)$ \\
\hline $2-4$ foci per $200 \times$ field & $7(23.3)$ \\
\hline$>4$ foci per $200 \times$ field & $0(0)$ \\
\hline \multicolumn{2}{|l|}{ Hepatocellular ballooning } \\
\hline None & $19(63.3)$ \\
\hline Few balloon cells & $9(10)$ \\
\hline Many balloon cells & $2(6.7)$ \\
\hline \multicolumn{2}{|l|}{ Fibrosis grade } \\
\hline None & $16(53.3)$ \\
\hline Perisinusoidal or periportal & $13(43.3)$ \\
\hline Perisinusoidal and periportal & $1(3.3)$ \\
\hline Bridging fibrosis & $0(0)$ \\
\hline Cirrhosis & $0(0)$ \\
\hline
\end{tabular}
time parameter

From Figure 2a and $\mathrm{c}$, it can be seen the relationship between mean calculated FIs and TR parameter. These 


\begin{tabular}{|c|c|c|c|c|}
\hline $\begin{array}{l}\text { Calculated FI } \\
\text { number }\end{array}$ & $\begin{array}{l}\text { Equation number } \\
\text { for estimation }\end{array}$ & $\begin{array}{l}\text { Sequence } \\
\text { number }\end{array}$ & Mean fat \pm SD (percentage) & Range (minimum-maximum) (\%) \\
\hline FI1* & Equ1** & Seq1 & $11.83 \pm 10.28$ & $-1.87-35.98$ \\
\hline FI2 & Equ1 & Seq2 & $15.02 \pm 12.02$ & $-1.31-40.84$ \\
\hline FI3 & Equ1 & Seq3 & $17.03 \pm 12.64$ & $-0.34-42.29$ \\
\hline FI4 & Equ1 & Seq4 & $17.59 \pm 12.7$ & $0.32-42.93$ \\
\hline FI5 & Equ1 & Seq5 & $10.03 \pm 9.25$ & $-2.13-32.44$ \\
\hline FI6 & Equ1 & Seq6 & $12.92 \pm 12.83$ & $-1.74-37.74$ \\
\hline FI7 & Equ1 & Seq7 & $15.04 \pm 12$ & $-1.61-41.27$ \\
\hline FI8 & Equ1 & Seq8 & $16.05 \pm 12.35$ & $-0.67-42.48$ \\
\hline FI9 & Equ1 & Seq9 & $9.22 \pm 8.9$ & $-2.49-30.87$ \\
\hline FI10 & Equ1 & Seq10 & $11.6 \pm 10.49$ & $-1.76-35.67$ \\
\hline FI11 & Equ1 & Seq11 & $13.66 \pm 11.42$ & $-1.76-39.51$ \\
\hline FI12 & Equ1 & Seq12 & $14.72 \pm 11.9$ & $-1.58-40.93$ \\
\hline FI13 & Equ1 & Seq13 & $9.42 \pm 8.97$ & $-1.92-31.23$ \\
\hline FI14 & Equ1 & Seq14 & $11.2 \pm 10.09$ & $-2-35.18$ \\
\hline FI15 & Equ1 & Seq15 & $13.18 \pm 11.18$ & $-1.37-39.07$ \\
\hline FI16 & Equ1 & Seq16 & $14.29 \pm 11.75$ & $-1.86-40.57$ \\
\hline FI17 & $\operatorname{Equ} 2 * * *$ & Seq1 & $-1.49 \pm 2.73$ & $-4.46-5.48$ \\
\hline FI18 & Equ2 & Seq2 & $0.25 \pm 4.34$ & $-5.18-10.33$ \\
\hline FI19 & Equ2 & Seq3 & $1.14 \pm 5.25$ & $-4.66-14.09$ \\
\hline FI20 & Equ2 & Seq4 & $2.09 \pm 5.81$ & $-5.88-14.7$ \\
\hline FI21 & Equ2 & Seq5 & $-2.33 \pm 1.98$ & $-4.84-2.83$ \\
\hline FI22 & Equ2 & Seq6 & $-0.98 \% \pm 3.15$ & $-4.77-6.7 \%$ \\
\hline FI23 & Equ2 & Seq7 & $-0.05 \pm 4.37$ & $-4.87-11.45$ \\
\hline FI24 & Equ2 & Seq 8 & $0.3 \pm 4.74$ & $-7-12.03$ \\
\hline FI25 & Equ2 & Seq9 & $-2.47 \pm 1.86$ & $-5.29-2.42$ \\
\hline FI26 & $\mathrm{Equ} 2$ & Seq10 & $-1.83 \pm 2.71$ & $-5.01-5.59$ \\
\hline FI27 & $\mathrm{Equ} 2$ & Seq11 & $-0.61 \pm 3.56$ & $-4.58-8.83$ \\
\hline FI28 & $\mathrm{Equ} 2$ & Seq12 & $-0.45 \pm 4.1$ & $-4.94-10.35$ \\
\hline FI29 & Equ2 & Seq13 & $-2.49 \% \pm 1.78$ & $-4.45-2.57$ \\
\hline FI30 & Equ2 & Seq14 & $-1.85 \pm 2.84$ & $-7.43-5.4$ \\
\hline FI31 & Equ2 & Seq15 & $-0.96 \pm 3.33$ & $-5.86-8.16$ \\
\hline FI32 & Equ2 & Seq16 & $-0.5 \pm 3.92$ & $-5.57-9.47$ \\
\hline FI33 & Equ3 & Seq1 & $17.85 \pm 10.68$ & $2.53-40.32$ \\
\hline FI34 & Equ3 & Seq2 & $20.92 \pm 11.97$ & $4.09-43.87$ \\
\hline FI35 & Equ3 & Seq3 & $22.95 \pm 12.27$ & $4.65-44.88$ \\
\hline FI36 & Equ3 & Seq4 & $23.58 \pm 12.22$ & $5.69-45.34$ \\
\hline FI37 & Equ3 & Seq5 & $16.13 \pm 9.84$ & $2.21-37.61$ \\
\hline FI38 & Equ3 & Seq6 & $18.97 \pm 11.02$ & $2.88-41.69$ \\
\hline FI39 & Equ3 & Seq7 & $21.09 \pm 11.91$ & $3.69-44.2$ \\
\hline FI40 & Equ3 & Seq 8 & $22.16 \pm 12.04$ & $5.07-45.09$ \\
\hline FI41 & Equ3 & Seq9 & $15.32 \pm 9.48$ & $2.67-36.45$ \\
\hline FI42 & Equ3 & Seq10 & $18.1 \pm 11.55$ & $2.97-48.94$ \\
\hline FI43 & Equ3 & Seq11 & $19.69 \pm 11.54$ & $3.45-42.97$ \\
\hline FI44 & Equ3 & Seq12 & $20.88 \pm 11.82$ & $4.03-43.96$ \\
\hline FI45 & Equ3 & Seq13 & $15.49 \pm 9.59$ & $2.13-36.76$ \\
\hline FI46 & Equ3 & Seq14 & $17.35 \pm 10.47$ & $2.54-39.69$ \\
\hline FI47 & Equ3 & Seq15 & $19.22 \pm 11.35$ & $2.02-42.54$ \\
\hline FI48 & Equ3 & Seq16 & $20.39 \pm 11.69$ & $3.34-43.68$ \\
\hline
\end{tabular}

*FI - Fat index calculated by MRI signal changes according to sequence number and equation; **Equ1=Equation 1: Equation for calculating FI by considering signal changes in first dual echoes Equation $1=\left(\frac{\mathrm{SI}_{\mathrm{IN}_{1}}-\mathrm{SI}_{\mathrm{OUT}_{1}}}{2 \mathrm{SI}_{\mathrm{IN}_{1}}}\right) \times 100 ; * * *$ Equ2 - Equation 2: Equation for calculating FI by considering signal changes in second dual echoes Equation 2=( $\left.\frac{\mathrm{SI}_{\mathrm{IN}_{2}}-\mathrm{SI}_{\mathrm{OUT}_{2}}}{2 \mathrm{SI}_{\mathrm{IN}_{2}}}\right) \times 100=; * * *$ Equ3 - Equation for calculating FI by considering signal changes at three first echoes Equation $3=\left(\frac{\left(\mathrm{SI}_{\mathrm{IN}_{1}} \exp ^{\left(4.86 / \mathrm{T}_{2}^{*}\right)}-\mathrm{SI}_{\mathrm{OUT}_{1}} \exp ^{\left(2.31 / \mathrm{T}_{2}^{*}\right)}\right)}{2 \mathrm{SI}_{\mathrm{IN}_{1}} \exp \left(4.86 / \mathrm{T}_{2}^{*}\right)}\right) \times 100$, Where $\mathrm{T}_{2}^{*}=\Delta \mathrm{TE} \ln \left(\frac{\mathrm{SI}_{\mathrm{IN}_{1}}}{\mathrm{SI}_{\mathrm{IN}_{2}}}\right) \cdot{ }^{[24,25]} \mathrm{MRI}^{*}-\mathrm{Magnetic}$ resonance imaging; FI - Fat index; SD - Standard deviation 

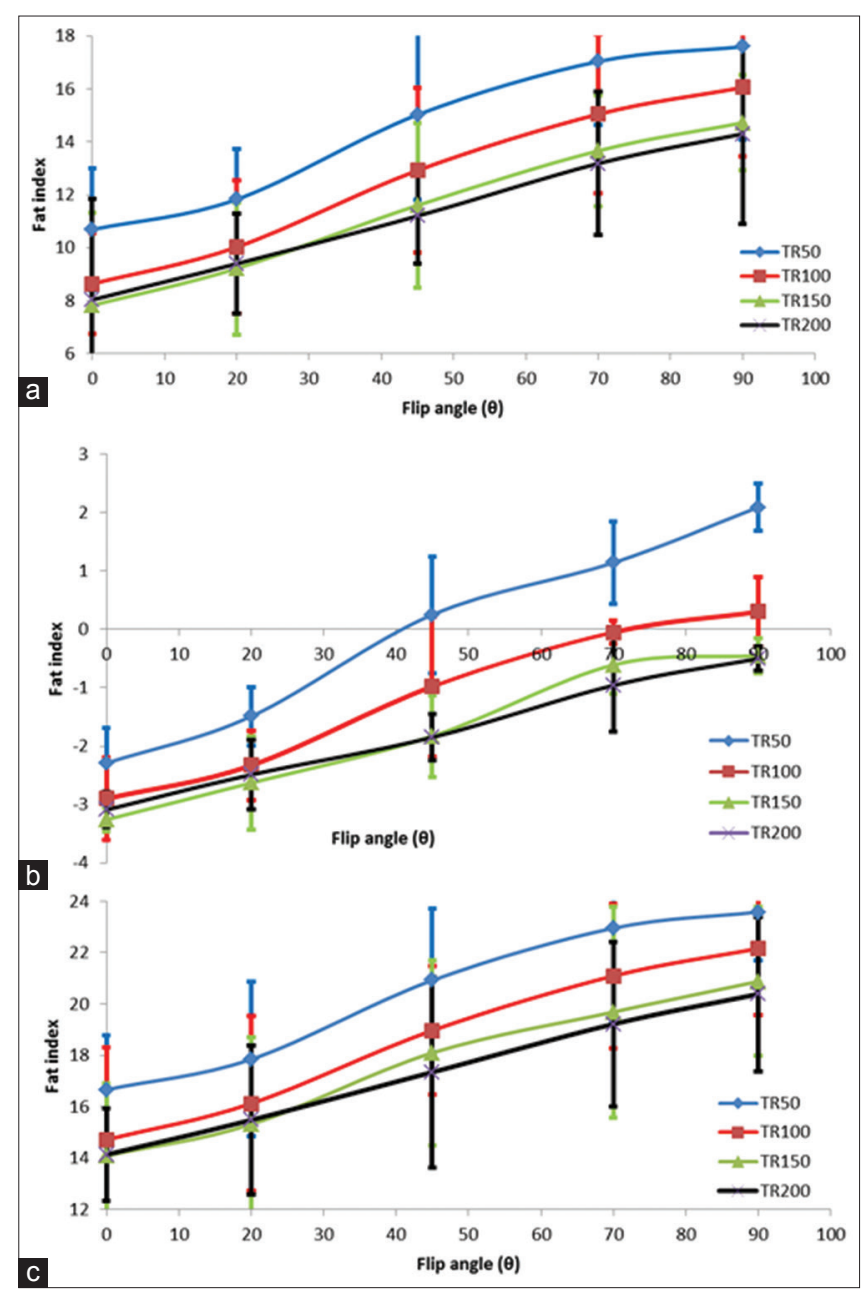

Figure 1: (a) Relationship between fat indexes calculated from Eq. 1, with flip angle at different repetition time. (b) Relationship between fat indexes calculated from Eq. 2, with flip angle at different repetition time. (c) Relationship between fat indexes calculated from Eq. 3, with flip angle at different repetition time

graphs show that the mean FI declines gradually with increasing TR parameter; however, as TR increases, calculated FI by method 2 [Figure $2 \mathrm{~b}$ ] decreased more intensely and irregularly [Figure 2].

\section{Correlational analysis}

Pearson's correlation coefficients were used to determine the relationship between obtaining various FIs by MR imaging and histopathologic liver steatosis. There is a strong correlation between all 48 FIs calculated with MR imaging and liver biopsy (correlation coefficients range from $r=0.81$ to $r=0.92$ ). The results of the correlation analysis are shown in Table 4 and all correlations are significant at the 0.01 level. It can be seen from the data in Table 4 that the correlations determined with the use of methods 1 and 3 were slightly stronger than the correlation with the use of method 2 in fat calculation at all sequences. Correlation coefficients corresponding to calculations with method 2 in all sequences were improved with a flip angle increasing $(r=0.82$ to
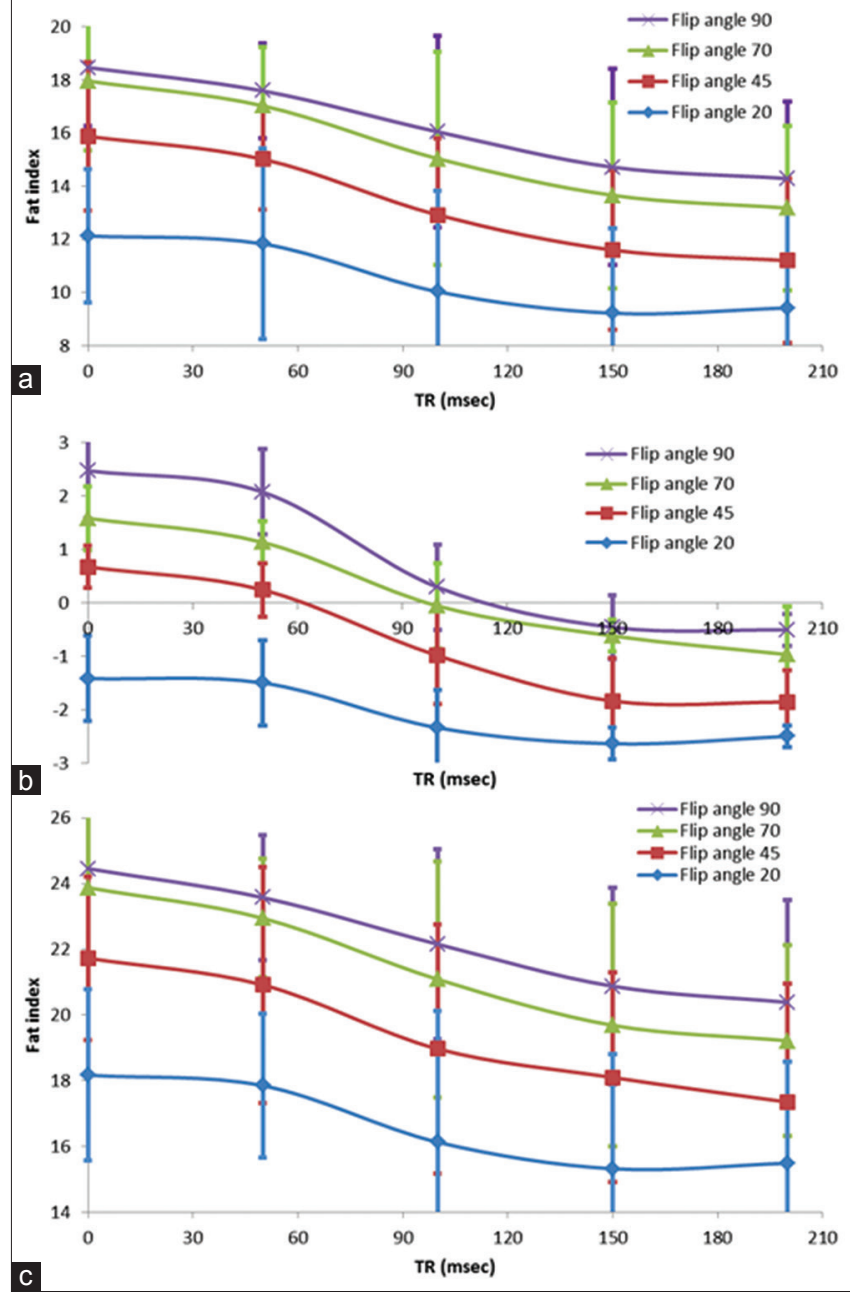

Figure 2: (a) Relationship between fat indexes calculated from Eq. 1, with repetition time parameter at different flip angles. (b) Relationship between fat indexes calculated from Eq. 2, with repetition time parameter at different flip angles. (c) Relationship between fat indexes calculated from Eq. 3, with repetition time parameter at different flip angle

$r=0.9$ ). From Table 4, FIs from sequence with small flip angle calculated with methods 1 and 3 have a slightly better correlation than FIs with large flip angle $(r=0.92$ in FI1 vs. $r=0.89$ in FI4) or ( $r=091$ in FI33 vs. $r=0.88$ in FI36) [Table 4].

\section{Discussion}

The present study was designed to investigate different chemical-shift GRE methods for fat quantifying in patients with NAFLD. Another more important question was to determine the effect of imaging parameters that control $T_{1}$ and $T_{2}^{*}$ relaxation in these methods. Our results demonstrate that first dual GRE techniques (method 1) and GRE techniques with correction of $T_{2}{ }^{*}$ decay effects (method 3) are better than second GRE techniques (method 2). Moreover, there was no significant difference observed between diagnostic accuracy of methods 1 and 3 in fat quantification. On the question of imaging parameters 


\begin{tabular}{|c|c|}
\hline Fat index & Pearson correlation coefficient with liver* \\
\hline 1 & 0.92 \\
\hline 2 & 0.91 \\
\hline 3 & 0.89 \\
\hline 4 & 0.89 \\
\hline 5 & 0.91 \\
\hline 6 & 0.91 \\
\hline 7 & 0.90 \\
\hline 8 & 0.90 \\
\hline 9 & 0.91 \\
\hline 10 & 0.91 \\
\hline 11 & 0.91 \\
\hline 12 & 0.91 \\
\hline 13 & 0.91 \\
\hline 14 & 0.91 \\
\hline 15 & 0.91 \\
\hline 16 & 0.91 \\
\hline 17 & 0.86 \\
\hline 18 & 0.87 \\
\hline 19 & 0.89 \\
\hline 20 & 0.87 \\
\hline 21 & 0.82 \\
\hline 22 & 0.88 \\
\hline 23 & 0.89 \\
\hline 24 & 0.90 \\
\hline 25 & 0.81 \\
\hline 26 & 0.86 \\
\hline 27 & 0.87 \\
\hline 28 & 0.90 \\
\hline 29 & 0.84 \\
\hline 30 & 0.83 \\
\hline 31 & 0.86 \\
\hline 32 & 0.90 \\
\hline 33 & 0.91 \\
\hline 34 & 0.90 \\
\hline 35 & 0.87 \\
\hline 36 & 0.88 \\
\hline 37 & 0.81 \\
\hline 38 & 0.90 \\
\hline 39 & 0.89 \\
\hline 40 & 0.89 \\
\hline 41 & 0.90 \\
\hline 42 & 0.90 \\
\hline 43 & 0.89 \\
\hline 44 & 0.91 \\
\hline 45 & 0.91 \\
\hline 46 & 0.91 \\
\hline 47 & 0.90 \\
\hline 48 & 0.90 \\
\hline
\end{tabular}

*All correlations are significant at the 0.01 level $(P<0 / 01)$

which affects fat measurement, this study found that flip angle parameter could be a major factor, if not the only one, causing the overestimation of liver fat content.
By referring to the data in Table 3 , maximum and minimum quantified FIs are related to the sequences with maximum and minimum $T_{1}$ weighting. From the literatures, phase interference is not only reason for using of GRE for fat quantification. Due to the shorter $T_{1}$ relaxation, signal of fat is higher at $T_{1}$ weighted sequences and leads to fat overestimation. ${ }^{[24]}$ These results are consistent with those of other studies and many previous authors suggest that to mitigate $T_{1}$ confounding effects, GRE sequences should be conducted with long TR and low flip angles. ${ }^{[26-28]}$

As shown in Table 3, most measured FIs with method 2, especially measured FIs in patients with low fat grades (FIs: 17-32), were negative. Signal loss due to $T_{2}{ }^{*}$ decay effects leads to more reduction of signal intensity at latter $\mathrm{IP}_{2}$ images relative to earlier $\mathrm{OP}_{2}$ images. As a consequence, FIs which calculated by method 2 have negative values. Furthermore, increasing of TE and $T_{2}{ }^{*}$ decay at last dual echoes causes that signal-to-noise ratio (SNR) diminished considerably. Thus, $\mathrm{OP}_{2}$ and $\mathrm{IP}_{2}$ images typically do not have an acceptable quality. Cassidy et al. ${ }^{[24]}$ recommended the use of first dual echoes to avoid this problem in practice.

Bydder et $a l{ }^{[22]}$ first explained the effect of flip angle for fat measurement on three NAFLD patients. Hansen et al. experimentally illustrated direct relationship between estimated fat and flip angle. ${ }^{[20]}$ Our findings are consistent with their studies and suggest that modifying flip angle affects $T_{1}$ weighting clearly and as a consequence measured FI. ${ }^{[22]}$ Theoretically, another parameter that controls $T_{1}$ weighting is TR. Increasing of TR parameter (slight decrease of $T_{1}$ weighting) decreases is calculated fat degree gradually. However, this parameter is not as effective as flip angle. Hansen et al ${ }^{[20]}$ findings show that the effect of TR on fat measurement did not statistically significant. In their study, TRs above $130 \mathrm{~ms}$ were examined. According to the results of this study, the amount of FIs remains constant at TRs above $100 \mathrm{~ms}$. The data reported here appear to support the assumption that the TRs above $100 \mathrm{~ms}$ do not have a significant effect on $T_{1}$ weighting contrast and determination of fat content.

Our findings show that the correlation coefficient of calculated FIs by methods 1 and 3 was more reliable than calculation by method 2. Weaker SNR at last echoes relative to first echoes could be the major reason. As in this method, correlation coefficients improve when flip angle increases (raising SNR). In accordance with the present study, some previous studies demonstrate that the correlation between calculated fat and biopsy findings was same with and without correction of $T_{2}{ }^{*}$ decay effects. ${ }^{[17,29]}$ Conversely our result is not in agreement with reported data by Westphalen et al that indicated the liver iron as a potential pitfall for fat quantification. ${ }^{[30]}$ This variation could be due to differences in the sample characteristics. Evidence from this study suggests that, in fat quantification with GRE techniques, it 
is possible that the third and fourth echoes are unnecessary. Furthermore, these last echoes subject to noise and maybe prolonged scan time and limit anatomy coverage.

Due to the nature of cross-sectional study, current investigation was limited by small sample size and caution must be applied, as the findings might not be transferable to NAFLD patients with fibrosis or iron deposition. Thus, more research will need to be done to determine the fat content in patients with these conditions.

\section{Conclusion}

$T_{1}$ relaxation effects probably more critical than $T_{2}^{*}$ to measurement of fat content. Our findings suggest that flip angle parameter could be a major factor, if not the only one, causing the overestimation of liver fat content. The sequences with low flip angle are more suitable for fat quantification. In this case, using of long TR is recommended to maintain SNR and image quality. However, increasing of scan time should be considered. The results of this research can be useful for adjustment of imaging parameter in GRE techniques to measurement of fat content.

\section{Acknowledgments}

This report was a part of Ph.D. Thesis results of the seventh author. The authors would like to thank all people who technically helped the work. The thesis was financially supported by Vice-Chancellor for Research Affairs of Ahvaz Jundishapur University of Medical Sciences (Grant number: RDC-9509).

\section{Financial support and sponsorship}

This study is funded by Vice-Chancellor for Research Affairs of Ahvaz Jundishapur University of Medical Sciences (Grant number: RDC-9509).

\section{Conflicts of interest}

There are no conflicts of interest.

\section{References}

1. Vernon G, Baranova A, Younossi ZM. Systematic review: The epidemiology and natural history of non-alcoholic fatty liver disease and non-alcoholic steatohepatitis in adults. Aliment Pharmacol Ther 2011;34:274-85.

2. Portillo-Sanchez $\mathrm{P}$, Bril F, Maximos $\mathrm{M}$, Lomonaco $\mathrm{R}$, Biernacki D, Orsak B, et al. High prevalence of nonalcoholic fatty liver disease in patients with type 2 diabetes mellitus and normal plasma aminotransferase levels. J Clin Endocrinol Metab 2015;100:2231-8.

3. Gorden DL, Myers DS, Ivanova PT, Fahy E, Maurya MR, Gupta S, et al. Biomarkers of NAFLD progression: A lipidomics approach to an epidemic. J Lipid Res 2015;56:722-36.

4. Chu MJ, Dare AJ, Phillips AR, Bartlett AS. Donor hepatic steatosis and outcome after liver transplantation: A Systematic review. J Gastrointest Surg 2015;19:1713-24.

5. Choi WT, Jen KY, Wang D, Tavakol M, Roberts JP, Gill RM, et al. Donor liver small droplet macrovesicular steatosis is associated with increased risk for recipient allograft rejection.
Am J Surg Pathol 2017;41:365-73.

6. Karlas T, Petroff D, Garnov N, Böhm S, Tenckhoff H, Wittekind $\mathrm{C}$, et al. Non-invasive assessment of hepatic steatosis in patients with NAFLD using controlled attenuation parameter and 1H-MR spectroscopy. PLoS One 2014;9:e91987.

7. Angulo P. Long-term mortality in nonalcoholic fatty liver disease: Is liver histology of any prognostic significance? Hepatology 2010;51:373-5.

8. Lee SS, Park SH. Radiologic evaluation of nonalcoholic fatty liver disease. World J Gastroenterol 2014;20:7392-402.

9. Nalbantoglu IL, Brunt EM. Role of liver biopsy in nonalcoholic fatty liver disease. World J Gastroenterol 2014;20:9026-37.

10. Ratziu V, Charlotte F, Heurtier A, Gombert S, Giral P, Bruckert E, et al. Sampling variability of liver biopsy in nonalcoholic fatty liver disease. Gastroenterology 2005;128:1898-906.

11. Ballestri S, Romagnoli D, Nascimbeni F, Francica G, Lonardo A. Role of ultrasound in the diagnosis and treatment of nonalcoholic fatty liver disease and its complications. Expert Rev Gastroenterol Hepatol 2015;9:603-27.

12. Schwenzer NF, Springer F, Schraml C, Stefan N, Machann J, Schick F, et al. Non-invasive assessment and quantification of liver steatosis by ultrasound, computed tomography and magnetic resonance. J Hepatol 2009;51:433-45.

13. van Werven JR, Marsman HA, Nederveen AJ, Smits NJ, ten Kate FJ, van Gulik TM, et al. Assessment of hepatic steatosis in patients undergoing liver resection: Comparison of US, CT, T1-weighted dual-echo MR imaging, and point-resolved $1 \mathrm{H}$ MR spectroscopy. Radiology 2010;256:159-68.

14. Guiu B, Petit JM, Loffroy R, Ben Salem D, Aho S, Masson D, et al. Quantification of liver fat content: Comparison of triple-echo chemical shift gradient-echo imaging and in vivo proton MR spectroscopy. Radiology 2009;250:95-102.

15. Hwang I, Lee JM, Lee KB, Yoon JH, Kiefer B, Han JK, et al. Hepatic steatosis in living liver donor candidates: Preoperative assessment by using breath-hold triple-echo MR imaging and $1 \mathrm{H}$ MR spectroscopy. Radiology 2014;271:730-8.

16. Satkunasingham J, Besa C, Bane O, Shah A, de Oliveira A, Gilson WD, et al. Liver fat quantification: Comparison of dual-echo and triple-echo chemical shift MRI to MR spectroscopy. Eur J Radiol 2015;84:1452-8.

17. Mennesson N, Dumortier J, Hervieu V, Milot L, Guillaud O, Scoazec JY, et al. Liver steatosis quantification using magnetic resonance imaging: A prospective comparative study with liver biopsy. J Comput Assist Tomogr 2009;33:672-7.

18. Borra RJ, Salo S, Dean K, Lautamäki R, Nuutila P, Komu M, et al. Nonalcoholic fatty liver disease: Rapid evaluation of liver fat content with in-phase and out-of-phase MR imaging. Radiology 2009;250:130-6.

19. Bydder M, Yokoo T, Hamilton G, Middleton MS, Chavez AD, Schwimmer JB, et al. Relaxation effects in the quantification of fat using gradient echo imaging. Magn Reson Imaging 2008;26:347-59.

20. Hansen KH, Schroeder ME, Hamilton G, Sirlin CB, Bydder M. Robustness of fat quantification using chemical shift imaging. Magn Reson Imaging 2012;30:151-7.

21. Hughes F, Bydder M, Middleton MS. Effects of T1 and T2 relaxation on liver fat quantification using GRE sequences. AJR Am J Roentgenol 2006;186:A57Y60.

22. Bydder M, Middleton MS, Chavez AD, Sirlin CB, editors. Effect of flip angle on fat quantification by Dixon techniques. In: Proceedings of the $14^{\text {th }}$ Annual Meeting of ISMRM, Seattle, WA, USA, 2006 (Abstract2300). 
23. Kleiner DE, Brunt EM, Van Natta M, Behling C, Contos MJ, Cummings OW, et al. Design and validation of a histological scoring system for nonalcoholic fatty liver disease. Hepatology 2005;41:1313-21.

24. Cassidy FH, Yokoo T, Aganovic L, Hanna RF, Bydder M, Middleton MS, et al. Fatty liver disease: MR imaging techniques for the detection and quantification of liver steatosis. Radiographics 2009;29:231-60.

25. Hussain HK, Chenevert TL, Londy FJ, Gulani V, Swanson SD, McKenna BJ, et al. Hepatic fat fraction: MR imaging for quantitative measurement and display - Early experience. Radiology 2005;237:1048-55.

26. Yokoo T, Bydder M, Hamilton G, Middleton MS, Gamst AC, Wolfson $\mathrm{T}$, et al. Nonalcoholic fatty liver disease: Diagnostic and fat-grading accuracy of low-flip-angle multiecho gradient-recalled-echo MR imaging at 1.5 T. Radiology 2009;251:67-76.
27. Hines CD, Yokoo $T$, Bydder $M$, Sirlin CB, Reeder SB Optimization of flip angle to allow tradeoffs in T1 bias and SNR performance for fat quantification. Proc Int Soc Magn Reson Med 2010;18:2927.

28. Meisamy S, Hines CD, Hamilton G, Sirlin CB, McKenzie CA, $\mathrm{Yu} \mathrm{H}$, et al. Quantification of hepatic steatosis with T1-independent, T2-corrected MR imaging with spectral modeling of fat: Blinded comparison with MR spectroscopy. Radiology 2011;258:767-75.

29. Kühn JP, Hernando D, Muñoz del Rio A, Evert M, Kannengiesser S, Völzke H, et al. Effect of multipeak spectral modeling of fat for liver iron and fat quantification: Correlation of biopsy with MR imaging results. Radiology 2012;265:133-42.

30. Westphalen AC, Qayyum A, Yeh BM, Merriman RB, Lee JA, Lamba A, et al. Liver fat: Effect of hepatic iron deposition on evaluation with opposed-phase MR imaging. Radiology 2007;242:450-5. 


\section{BIOGRAPHIES}

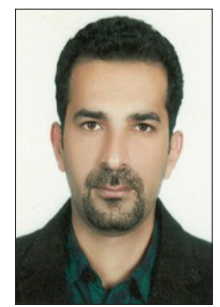

Mansour Zabihzadeh received his B.Sc degree in physics in 2000 from Shahid Rajaee University of Karaj, Karaj, Iran; and he obtained his Master and PhD degree in medical physics from Tehran University of medical Sciences, Tehran, Iran in 2003 and 2009 respectively, he is now an associated professor at Ahvaz Jundishapur University of medical Sciences. His research interest include Radiotherapy, radiation dosimetry, Image analysis.

Email: manzabih@gmail.com

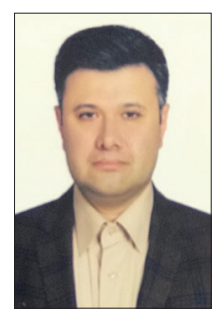

Mohammad Momen Gharibvand

received his MD degree from Shiraz University of medical Sciences, Shiraz, Iran, in 1999 and his radiology Specialty from Shahid Beheshti University of medical Sciences, Tehran, Iran in 2005, he is currently assistant professor at Ahvaz Jundishapur University of medical Sciences, His research interests include intervention radiology, image analysis, quantitative imaging.

Email: mohamad.momen2017@gmail.com

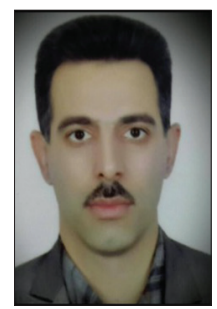

Azim Motamedfar received his MD degree from Tabriz University of medical Sciences, Tabriz, Iran, in 2001 and his radiology Specialty from Tehran University of medical Sciences, Tehran, Iran in 2006, he is currently assistant professor at Ahvaz Jundishapur University of medical Sciences, His research interests include intervention radiology, image analysis, quantitative imaging.

Email: AZEXIOUS@yahoo.com

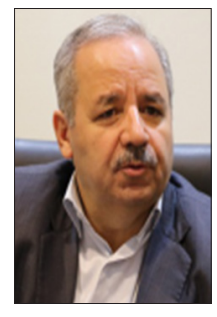

\section{Morteza Tahmasebi}

received his MD and radiology Specialty degree from Tehran University of medical Sciences, Shiraz, Iran, in 1989 and 2005, respectively,he is currently associated professor at Ahvaz Jundishapur University of medical Sciences, His research interests include intervention radiology, dynamic imaging, quantitative imaging.

Email: tahmasebilmn@yahoo.com

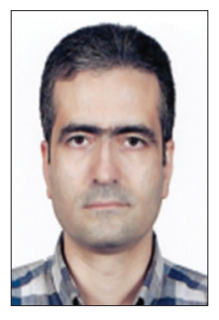

Amir Hossein Sina received his MD degree from Mashhad University of medical Sciences, Mashhad, Iran, in 2004 and his pathlogy Specialty from Iran University of medical Sciences, Tehran, Iran in 2010, he is currently assistant professor at Ahvaz Jundishapur University of medical Sciences, His research interests in the field of pathlogy disease assessment.

Email: dramirhoseinsina@yahoo.com

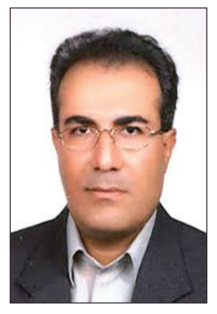

Kavous Bahrami received his B.Sc degree in radiology technology from Ahvaz Jundishapur University of medical Sciences, Ahvaz, Iran, in 1998, He is currently working at the MRI department of Golestan hospital, Ahvaz, Iran. his research interests are in the fields of imaging technology.

Email: kavous_bahrami@yahoo.com

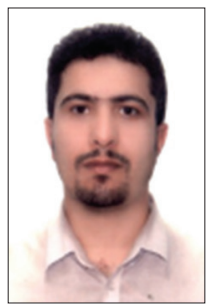

Mozafar Naserpour received his B.Sc degree in radiology technology from Tehran University of medical Sciences, Tehran, Iran in 2005, and he got his M.Sc and $\mathrm{PhD}$ degrees from Ahvaz Jundishapur University of medical Sciences in 2011 and 2018 respectively, he is now an assistant professor at Behbahan Faculty of medical Sciences, his research interests are in the fields of imaging technology, radiation dosimetry, signal processing. Quantitative MRI.

Email: naserpour1983@gmail.com 